\title{
Autophagy for better or worse during infectious diseases
}

\section{Irina Caminschi ${ }^{1}$ and Christian Münz ${ }^{2 *}$}

1 Centre for Immunology, Burnet Institute, Melbourne, VIC, Australia

2 Institute of Experimental Immunology, University of Zürich, Zürich, Switzerland

*Correspondence: christian.muenz@uzh.ch

\section{Edited by:}

Christian Kurts, Friedrich Wilhelms-Universität Bonn, Germany

Autophagy describes at least three metabolic pathways that deliver cytoplasmic constituents for lysosomal degradation (1). While micro- and chaperone-mediated autophagy engulf or translocate cytosolic material at the late endosomal or lyosomal membrane, respectively, macroautophagy can use different membrane sources, including endoplasmic reticulum, Golgi, plasma membrane, mitochondria, and outer nuclear membrane to enclose large portions of the cytoplasm in autophagosomes (2). These double membrane surrounded vesicles are generated de novo around macroautophagy cargo like damaged organelles, protein aggregates, and cytosolic pathogens, and more than 30 autophagy related (Atg) gene products are involved in their formation and fusion with lysosomes. The series of review articles in this Frontiers in Immunologye-book will high-light how regulation of macroautophagy during infections results in cytosolic restriction of pathogens, sometimes supports their replication and is connected to innate immune activation as well as adaptive immune responses to these environmental insults.

In the first set of reviews, the interactions of pathogens with macroautophagy will be discussed. Dengjel and coworkers will summarize the regulation of macroautophagy by influenza A virus and how this changes macroautophagic flux (3). This review particularly focuses on the sequential recruitment of substrates to autophagosomes and interference by influenza A virus. A second review by Biard-Piechaczyk and coauthors will then discuss the different functions that macroautophagy has during human immunodeficiency virus (HIV) infection of T cells and macrophages (4). Differences in the viral replication within these two host cells appear to determine the role that macroautophagy plays in HIV propagation in these targets. Furthermore, Faure and coworkers will high-light that there are certain nodes in the macroautophagy network that are targeted by many viruses (5). Particularly the GTPase IRGM will be discussed. Moreover, Taylor and colleague will discuss the regulation of macroautophagy by herpesviruses (6). Atg6/ Beclin-1 targeting by these pathogens has resulted in fascinating insights and tools to dissect macroautophagy. Finally, this block of reviews is concluded with a text by Sasakawa and coworkers (7). They discuss the restriction of bacterial dissemination by macroautophagy and the counter responses of the bacteria aimed at escaping these immune measures. Thus, many pathogens regulate and are restricted by macroautophagy during infection.

A second set of reviews explores the role of macroautophagy in immune responses. Innate immune recognition, resulting cytokine production, antigen processing for $\mathrm{MHC}$ presentation, and autoimmunity will be discussed in this block. Lee and coworkers will discuss how macroautophagy regulates pathogen detection by the immune system (8). Both the turnover of cytosolic receptors of pathogen associated molecular patterns (PAMPs) and the transport of PAMPs to vesicular receptors is affected by macroautophagy. Moreover, early innate cytokine production is regulated by this pathway. Harris discusses the influence of macroautophagy on IL-1 production (9). Furthermore, Villadangos and colleague highlight the role of macroautophagy in innate and adaptive immunity, covering its role in antigen processing, as well as in T and B cell physiology (10). Expanding on some of these themes, Albert and co-worker summarize the evidence that macroautophagy contributes to exogenous antigen cross-presentation onto MHC class I molecules (11) and focus on the role of the antigen donor cell. In a second review on antigen processing via macroautophagy, the role of this pathway in MHC class II antigen processing will be discussed (12). Particularly, its contribution to both intracellular and extracellular antigen processing will be considered. Beyond antigen processing, $\mathrm{He}$ and colleagues will review the role for macroautophagy in lymphocyte development and function (13). This article focuses on the role of macroautophagy in T cells. Finally, Eissa and coauthor summarize how macroautophagy alterations might lead to hyperreactivity to gut commensals and autoimmunity (14). In this respect genetic predisposition to Crohn's disease, which affects essential autophagy genes, will be discussed. Thus, this second set of reviews captures the breadth of functions for macroautophagy in immunity.

Macroautophagy is, therefore, not only an essential metabolic pathway, but has also been used during the co-evolutionary struggle between pathogens and their hosts to benefit one or the other. One would predict that it may also play a role in many other infectious diseases, and consequently, could serve as a therapeutic target. However, since macroautophagy can serve the immune system or be exploited by the pathogen, its role has to be characterized for every single different pathogen in order to predict the effect its manipulation would have during infection.

\section{REFERENCES}

1. Mizushima N, Yoshimori T, OhsumiY. The role of Atg proteins in autophagosome formation. Annu Rev Cell Dev Biol (2011) 27:107-32. doi: 10.1146/ annurev-cellbio-092910-154005

2. Rubinsztein DC, Shpilka T, Elazar Z. Mechanisms of autophagosome biogenesis. Curr Biol (2012) 22:R29-34. doi: 10.1016/j.cub.2011.11.034

3. Dumit VI, Dengjel J. Autophagosomal protein dynamics and influenza virus infection. Front Immunol (2012) 3:43. doi: 10.3389/fimmu.2012.00043

4. Borel S, Espert L, Biard-Piechaczyk M. Macroautophagy regulation during HIV-1 infection of CD4 ${ }^{+} \mathrm{T}$ cells and macrophages. Front Immunol (2012) 3:97. doi: 10.3389/fimmu.2012.00097

5. Petcova DS, Viret C, Faure M. IRGM in autophagy and viral infections. Front Immunol (2013) 3:426. doi: 10.3389/fimmu.2012.00426

6. Williams LR, Taylor GS. Autophagy and immunity - insights from human herpesviruses. Front Immunol (2012) 3:170. doi: 10.3389/fimmu.2012.00170 
7. Jo EK, Yuk JM, Shin DM, Sasakawa C. Roles of autophagy in elimination of intracellular bacterial pathogens. Front Immunol (2013) 4:97. doi: 10.3389/ fimmu.2013.00097

8. Oh JE, Lee HK. Modulation of pathogen recognition by autophagy. Front Immunol (2012) 3:44. doi: 10.3389/fimmu.2012.00044

9. Harris J. Autophagy and IL-1 family cytokines. Front Immunol (2013) 4:83. doi: 10.3389/fimmu.2013.00083

10. Mintern JD, Villadangos JA. Autophagy and mechanisms of effective immunity. Front Immunol (2012) 3:60. doi: 10.3389/fimmu.2012.00060

11. Joubert PE, Albert ML. Antigen cross-priming of cell-associated proteins is enhanced by macroautophagy within the antigen donor cell. Front Immunol (2012) 3:61. doi: 10.3389/fimmu.2012.00061

12. Münz C. Antigen processing for MHC class II presentation via autophagy. Front Immunol (2012) 3:9. doi: 10.3389/fimmu.2012.00009
13. He MX, Mcleod IX, Jia W, He YW. Macroautophagy in T lymphocyte development and function. Front Immunol (2012) 3:22. doi: 10.3389/fimmu.2012.00022

14. Bhattacharya A, Eissa NT. Autophagy and autoimmunity crosstalks. Front Immunol (2013) 4:88. doi: 10.3389/fimmu.2013.00088

Received: 27 June 2013; accepted: 06 July 2013; published online: 19 July 2013. Citation: Caminschi I and Münz C (2013) Autophagy for better or worse during infectious diseases. Front. Immunol. 4:205. doi: 10.3389/fimmu.2013.00205

This article was submitted to Frontiers in Antigen Presenting Cell Biology, a specialty of Frontiers in Immunology.

Copyright $\odot 2013$ Caminschi and Münz. This is an open-access article distributed under the terms of the Creative Commons Attribution License, which permits use, distribution and reproduction in other forums, provided the original authors and source are credited and subject to any copyright notices concerning any third-party graphics etc. 